\title{
Una descripción y caracterización del Programa de Transferencias Condicionadas “Ciudadanía Porteña - Con todo derecho"."
}

A description and characterization of the Conditional Cash Transfer Program "Porteña Citizenship".

\author{
Melina Ayelén Patti ${ }^{* *}$
}

\section{Resumen}

El presente artículo se propone contribuir a una caracterización del Programa de Transferencias Condicionadas de Ingreso "Ciudadanía Porteña", en tanto política social desarrollada en el ámbito de la Ciudad Autónoma de Buenos Aires. Para cumplir este objetivo se describen, sintéticamente, las principales dimensiones que construyen la matriz del Programa, haciendo énfasis en el relevamiento de aspectos vinculados a sus contraprestaciones en salud y educación.

\section{Palabras clave}

Políticas Sociales, Programas de Transferencia Condicionada de Ingresos, Contraprestaciones.

\footnotetext{
* Artículo recibido el 1 de octubre de 2015. Aceptado el 9 de Diciembre de 2015.

** Licenciada en Ciencias de la Comunicación - Universidad de Buenos Aires

Correo electrónico: melina.patti@gmail.com
} 


\section{Abstract}

The purpose of this article is to contribute shaping a characterization of the Conditional Cash Transfer Program "Porteña Citizenship", as social policy is developed in the area of Buenos Aires Autonomous City. To reach this aim we describe, briefly, the main aspects that build up the head of the program, focused on the survey of health and education conditions.

\section{Keywords}

Social Policies, Programs of conditional cash transfer,

A finales de la década de 1990, la realidad socioeconómica de los países latinoamericanos, producto de la apertura de las economías; la desregulación, o, en términos de Basualdo y Azpiazu (2004), los procesos de 're-regulación' del mercado tendientes a transferir un mayor poder regulatorio al capital, promovidos por la acción u omisión estatal y la disolución del mercado de trabajo, hizo sumergir a estos países en profundas crisis, procesos recesivos, escenarios de vulnerabilidad social y un marcado deterioro de las condiciones de vida de los hogares más pobres. Frente a esta situación, las políticas sociales debieron asumir un rol clave en lo inmediato.

En este contexto, surgieron las primeras implementaciones en la región de América Latina y el Caribe de los Programas de Transferencia Condicionada de Ingresos impulsados por los principales organismos multilaterales de crédito, primero en Brasil

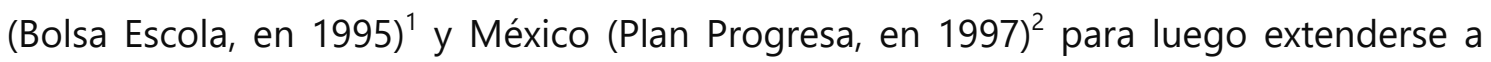
otros países de América del Sur y el Caribe.

Recién a principios del nuevo milenio, estas políticas públicas comenzaron a constituirse en opciones de protección social ampliando su cobertura como parte de

\footnotetext{
${ }^{1}$ En la actualidad devino en un programa de cobertura más amplia con el nombre de Bolsa Familia.

2 En marzo de 2002 PROGRESA cambió el nombre a Desarrollo Humano Oportunidades, e introdujo varios cambios a sus objetivos y características operativas, incluyendo su expansión a áreas urbanas. En septiembre de 2014 se modificó nuevamente para dar paso al programa PROSPERA, el cual constituye una nueva fase de Oportunidades.
} 
las estrategias de reducción de la pobreza y desigualdad social, con el objetivo de fortalecer el capital humano de la población destinataria.

El objetivo del artículo es revisar la matriz que configura un Programa de Transferencias Condicionadas de Ingreso (en adelante, PTCI), en particular, el caso del Programa "Ciudadanía Porteña-Con todo derecho" de la Ciudad Autónoma de Buenos Aires, luego de transcurrida una década de su implementación. ${ }^{3}$

La estrategia expositiva seleccionada ha sido la siguiente: en primer lugar, se definen las políticas sociales y se sintetiza el punto de partida teórico; en segundo lugar, se realiza una breve historización de los PTCl en América Latina y en Argentina, para luego conceptualizarlos; en tercer lugar, se explicita la estrategia metodológica del trabajo; en cuarto lugar se describe y caracteriza el programa "Ciudadanía Porteña" en base a dimensiones de análisis construidas para su indagación; por último, se ensayan las reflexiones finales con el objetivo de abrir algunos interrogantes sobre el tema.

Este trabajo inicia su recorrido desde una mirada que define las políticas sociales estrechamente asociadas a los procesos y estrategias de acumulación de capital, en tanto es política no solo por el recorte temático que le es asignado, sino por su inscripción en una determinada estructura de dominación social y una particular configuración del régimen político.

En este sentido, Laurell (citado en Vilas, 1995: 186) señala que "... las alternativas de política social no se debaten principalmente en el terreno técnico (...) sino en el ámbito de los proyectos políticos. Obedecen a concepciones distintas sobre cómo organizar la sociedad y en función de qué intereses satisfacer necesidades". Siguiendo esta línea, se afirma que la política social consiste en el conjunto más o menos coherente de principios y acciones gestionadas por el Estado, que determinan la distribución y control social del bienestar de una población por (la) vía política (Herrara y Castón, 2003).

Asimismo, las políticas sociales son prácticas estatales que delinean lo social al tener la capacidad de construir realidades. El Estado se posiciona como un actor -y un ámbito- en la producción y reproducción de los problemas sociales, en la delimitación de sus responsabilidades, en la definición de los sujetos merecedores de sus intervenciones y de las condiciones para dicho merecimiento. Dichas políticas públicas "... hacen sociedad al actuar sobre y ser resultado de los modelos de estructuración y acumulación social, al mismo tiempo operan sobre los aspectos simbólicos de la vida...". (De Sena y Mona, 2014: 13)

\footnotetext{
${ }^{3}$ El presente artículo forma parte de la tesina de grado "Programas de Transferencia Condicionada de Ingresos, Comunicación y Salud" realizada en el marco del último ciclo de la Licenciatura en Ciencias de la Comunicación en la Universidad de Buenos Aires. Dicho trabajo de investigación tuvo como finalidad indagar acerca de la matriz comunicacional de $\mathrm{CP}$ en relación con sus requisitos (contraprestaciones) en salud.
} 
Las transformaciones experimentadas en el modelo de acumulación de capital a partir de la década de 1980 en Latinoamérica tuvieron como consecuencia cambios de equivalente magnitud y proyecciones en el terreno de la política social. Los procesos de desarticulación del mercado laboral, con el efecto del crecimiento de los niveles de desempleo y subempleo, la precarización, el deterioro de los ingresos de los trabajadores, los procesos de desindustrialización y la apertura indiscriminada de las economías alimentaron el crecimiento de la magnitud de la pobreza y las desigualdades sociales.

En términos generales, y retomando la definición propuesta por Cecchini y Madariaga $(2011)^{4}$ la principal característica de la mayoría los PTCI consiste en la entrega de recursos monetarios a familias en situación de pobreza que tienen uno o más hijos menores de edad y a mujeres embarazadas, con la condición de que cumplan con ciertos compromisos (condicionalidades) requeridos, que se relacionan principalmente con las áreas de educación, salud y nutrición (por ejemplo, que los niños concurran a la escuela o a los centros de salud con fines preventivos y de atención primaria). En este punto, interesa resaltar que este tipo de políticas sociales establecen a priori la titularidad femenina. Ello se encuentra estrechamente relacionado con el rol que se otorga a las mujeres en el ámbito doméstico como organizadoras del cuidado. La mujer-madre es la principal responsable de llevar a cabo las contraprestaciones que exigen los programas.

Asimismo, los PTCl adoptan criterios técnicos para la selección de los destinatarios a través de la focalización según unidades geográficas y condiciones de vida.

Las contraprestaciones/condicionalidades que caracterizan a los PTCl dan cuenta de una visión de la pobreza entendida como carencia de activos y oportunidades, cuya resolución puede encontrarse en la inversión en el capital humano de los pobres (Puello Socarrás; Gunturiz, 2013).

De acuerdo al balance de la experiencia en América Latina y el Caribe de los PTCl realizado por Cecchini y Madariaga (2011) según la base de Programas de Protección social no contributiva en América Latina y el Caribe de la CEPAL, los PTCI "... operan en la actualidad en 18 países de la región y benefician a más de 25 millones de familias (alrededor de 113 millones de personas), es decir, el 19\% de la población de

\footnotetext{
${ }^{4}$ Este documento ha sido preparado por Simone Cecchini, Oficial de Asuntos Sociales de la División de Desarrollo Social de la Comisión Económica para América Latina y el Caribe (CEPAL), y Aldo Madariaga, asistente de investigación, en el marco del componente Asistencia social: reducción de la pobreza y redistribución del ingreso a través de los programas de transferencias condicionadas, del programa de cooperación de la CEPAL y la Agencia Sueca de Cooperación Internacional para el Desarrollo (Asdi) 20102011, Protección e inclusión social en América Latina y el Caribe.
} 
América Latina y el Caribe, a un costo que ronda el 0,4\% del PIB regional" (Cecchini y Madariaga, 2011: 7).

En Argentina, se desarrolló la primera experiencia masiva de PTCl con el Plan Trabajar (1996), el cual proveía a jefes de familia con necesidades básicas insatisfechas una ocupación transitoria en el área de la construcción y el mantenimiento de infraestructura comunitaria.

A raíz de la crisis económica y social que atravesó el país en el año 2001-2002 y la profundidad del deterioro de la situación social, el gobierno nacional implementó, en el año 2002, el programa Jefes y Jefas de Hogar Desocupados (PJJHD). Este programa de transferencias de ingresos se caracterizó por su extensa cobertura -alcanzó a cubrir casi 2 millones de hogares, el 20\% de los existentes en el país- e incorporó la introducción de contraprestaciones, tanto laborales (como en el Plan Trabajar) como educativas (Cruces, Epele y Guardia, 2008).

Para el año 2006, se inició una estrategia gradual de salida del PJJHD mediante la creación de dos programas: el Seguro de Capacitación y Empleo (SCyE) y el Programa Familias por la Inclusión Social (denominado habitualmente Plan Familias). Este último constituyó una transferencia monetaria a hogares -con determinadas características- por cada menor de 18 años a cargo, bajo la condición de cumplir con su educación y salud.

En la actualidad, funcionan a nivel nacional la Asignación Universal por Hijo para Protección social (2009) y la Asignación por embarazo para Protección Social (2011) bajo la dependencia directa la Administración Nacional de la Seguridad Social (ANSES). ${ }^{5}$ Esta política establece un ingreso por hijo (hasta cinco) menor de 18 años a los núcleos familiares que se encuentren desempleados o desarrollando empleos en el sector de la economía informal, en tanto los hogares deben presentar los certificados de cumplimiento con el plan nacional de vacunación y controles médicos estipulados de los hijos menores a cargo y durante el embarazo, y el cumplimiento con la asistencia escolar obligatoria.

A nivel local, en la Ciudad Autónoma de Buenos Aires (en adelante, C.A.B.A) se desarrollan, en la actualidad, el programa Ciudadanía Porteña-Con todo derecho (2005) y el programa Ticket Social (2008) dependientes del Ministerio de Desarrollo Social de la Ciudad de Buenos Aires.

Este artículo presenta una descripción y caracterización del programa Ciudadanía Porteña-Con todo derecho (en adelante, CP) tras una década de implementación. Como estrategia metodológica, se trabajó con datos secundarios, a fin

\footnotetext{
${ }^{5}$ Cabe señalar que para algunas autoras la AUH no resulta un PTC (Pautassi, Arcidiácono, Straschnoy, 2013 entre otros), en tanto Repetto y Dalmasetto (2011) consideran que se integra a los Programas de Transferencia Condicionada.
} 
de buscar reconstruir y describir, a través del análisis de la información oficial producida por distintos organismos estatales, el modo en que se han definido, diseñado y desarrollado este tipo de intervenciones en la C.A.B.A.

El desarrollo de este trabajo se realizó a través de la recopilación documental mediante la revisión del sitio Web de C.A.B.A. , de la Ley 1878/2005 de la Legislatura de C.A.B.A. , y el análisis de datos provenientes de fuentes secundarias: indicadores sociales de C.A.B.A provenientes del Censo Nacional de Población, Hogares y Viviendas (2010), Encuesta Anual de Hogares (2014) y Encuesta Trimestral de Ocupación e Ingresos (2014), Estadísticas vitales y Análisis de condiciones de salud de C.A.B.A, Informes de evaluación del programa Ciudadanía Porteña del Área, Monitoreo y Evaluación de la Subsecretaria de Administración, Ministerio de Desarrollo Social, Encuesta a Beneficiarios de CP (2011) y el Monitoreo mensual de mayo del programa Ciudadanía Porteña del Área Monitoreo y Evaluación, Subsecretaría de Administración, Ministerio de Desarrollo Social (2015). El relevamiento de la información consignada tiene como fecha de inicio el mes de agosto del año 2014 y de corte, el mismo mes del año 2015.

Para organizar el análisis, el artículo identifica y analiza las dimensiones que configuran la política social y permiten construir una aproximación a las características y particularidades de CP: población objetivo; indicadores sociodemográficos de los hogares y características de los titulares; proceso de inscripción al programa; mecanismos de selección de los beneficiarios; modalidad y monto del beneficio; obligaciones en salud y educación (contraprestaciones); incorporación de subprogramas complementarios de CP; impacto sobre los indicadores sociales y la educación; y, por último, impactos sobre indicadores de salud.

Dichas dimensiones permiten echar luz sobre la matriz conceptual desde donde se concibe este tipo de políticas sociales, y abordar su diseño e implementación, permitiendo describir los objetivos, el alcance, los destinatarios, los actores, la institucionalidad, las prioridades de la política social y la dimensión política de los sistemas de protección social.

El programa Ciudadanía Porteña-Con todo derecho es un Programa de Transferencias Condicionadas de Ingreso o con corresponsabilidad (PTCl) que se ejecuta desde el año 2005 en el ámbito del Ministerio de Desarrollo Social del Gobierno de la Ciudad de Buenos Aires. Este se inscribe dentro de las políticas de protección social no contributiva, un conjunto de programas de transferencias monetarias y subsidios públicos financiados mediante impuestos a los ingresos generales bajo el principio de solidaridad.

CP fue creado por la Ley 1878/2005 de la Legislatura de C.A.B.A. y es gestionado por la Dirección General de Ciudadanía Porteña (instituida en 2006), que actualmente depende de la Subsecretaria de Fortalecimiento Familiar y Comunitario a cargo del Ministerio de Desarrollo Social. 
Sus antecedentes inmediatos son el programa Vale Ciudad (programa de entrega de vales para ser canjeados por alimentos en una red de comercios minoristas que se efectuaba en zonas específicas y determinadas de C.A.B.A.) y el programa Apoyo Alimentario Directo a Familias, creados en 2001 y 2002 (Asesoría General Tutelar, 2011). De acuerdo a la Cláusula Transitoria de la Ley 1878 de creación del programa $C P$, este absorbió a los beneficiarios de los programas alimentarios Vale Ciudad y Apoyo Alimentario, en tanto calificaran como población beneficiaria, conforme al artículo $4^{\circ}$ de la ley.

CP fue diseñado con el objetivo de disminuir los niveles de desigualdad en C.A.B.A. e interrumpir los circuitos de reproducción intergeneracional de la pobreza, efectuando una transferencia de ingresos a los integrantes de las familias de menores recursos. Se propone "sostener el acceso a la alimentación de la población beneficiaria, así como promover el acceso a la educación y protección de la salud de los niños, niñas, adolescentes y su grupo familiar, la búsqueda de empleo y reinserción en el mercado laboral de los adultos" (Ley No 1878. Artículo 2, 2005).

Se trata de una prestación monetaria mensual que tiene como contraprestación, en el caso de las familias con hijos, el cumplimiento de ciertos requisitos en educación y en materia de salud materno-infanto-juvenil. ${ }^{6}$

\section{La construcción de la población destinataria de CP (población objetivo)}

El programa dirige sus acciones a los hogares residentes en C.A.B.A. en situación de pobreza e indigencia, ${ }^{7}$ enfatizando su accionar en los de mayor vulnerabilidad. Entre estas características se destacan: la presencia de embarazadas, menores de 18 años de edad, discapacitados y adultos mayores.

De acuerdo al artículo 4 de la Ley $N^{\circ} 1878$, son destinatarios del programa, en el siguiente orden de prioridad y conforme la aplicación del índice de vulnerabilidad:

1) Hogares cuyos ingresos resultan hasta un $25 \%$ por encima de la línea de indigencia.

2) Hogares cuyos ingresos resultan superiores al previsto en el inciso anterior y hasta la línea de pobreza, con hijos a cargo de hasta 18 años cumplidos y/o mujeres embarazadas y/o adultos mayores 65 años a cargo y/o personas con necesidades especiales a cargo.

\footnotetext{
${ }^{6}$ Fuente: Página web del Gobierno de la Ciudad de Buenos Aires - Ministerio de Desarrollo Social Ciudadanía Porteña: http://www.buenosaires.gob.ar/areas/des_social/ciudadania_portenia

${ }^{7}$ El programa considera 'hogares pobres' a aquellos que se encuentran bajo la Línea de Pobreza (LP) que elabora el Instituto Nacional de Estadística y Censos (INDEC). Los 'hogares indigentes' son aquellos cuyo ingreso no supera la Canasta Básica Alimentaria (CBA).
} 
3) Hogares con ingresos superiores a los indicados en el inciso a) y hasta la línea de pobreza, sin hijos a cargo de hasta 18 años, sin mujeres embarazadas, que no tienen adultos mayores 65 años ni personas con discapacidad a cargo, según el grado de intensidad en función de la demanda efectiva para este programa (Ley $N^{\circ} 1878$. Artículo 4, 2005).

Para acceder a $\mathrm{CP}$, es necesario presentar el documento nacional de identidad argentino de todas las personas que conforman el hogar, residencia en C.A.B.A no menor a dos años, el CUIL de las personas mayores de 18 años, partida de nacimiento de los menores, certificados de estado de salud y certificados de educación de todos los integrantes del hogar menores a 18 años.

Los hogares cuyos miembros mayores de 18 años al momento de la inscripción no cuentan con documentación argentina deben realizar una preinscripción al programa; el trámite de inscripción se debe completar al regularizar su situación migratoria; lo mismo para el resto de los integrantes del hogar, que para poder ser incluidos en la solicitud deben iniciar la tramitación del documento argentino que acredite identidad. ${ }^{8}$

La focalización que tiene el programa CP no considera la inserción laboral en el mercado de trabajo formal o la percepción o no de jubilación o pensión una exclusión para obtener la titularidad del beneficio, como sí lo hacen otros sistemas de protección social como la Asignación Universal por Hijo (AUH).

La descripción de la población destinataria realizada a través de la normativa del programa permite señalar el establecimiento de prioridades en términos de situación de pobreza y vulnerabilidad, explicitando distintos tipos o grados de pobreza. Es decir, $\mathrm{CP}$ está dirigido a un tipo de población particular, señalando que no es para todos y dejando en evidencia su focalización.

Este tipo de políticas focalizadas que presentan su particularidad en el número de personas que perciben el ingreso, constituye, como sostiene De Sena (2011) una "focalización ocluida", ya que, en tanto programas masivos, se presentan como algo 'para muchos' pero que no incluye a todos. Se deja a una parte de la población total excluida, habilitando la persistencia de criterios rectores para la 'elegibilidad' del sujeto.

\section{Descripción sociodemográfica de los hogares}

De acuerdo a los resultados del Informe de Monitoreo Ciudadanía Porteña realizado en mayo de 2015 por la Gerencia Operativa de Gestión Estratégica de

\footnotetext{
8 El pprograma Patria Grande ha agilizado el proceso para las personas nacidas en los países del MERCOSUR y Estados asociados. Se trata del Programa Nacional de Normalización Documentaria Migratoria que entró en vigencia el 17 de abril de 2006. Está destinado a los ciudadanos nativos de países miembros del Mercosur y de los Estados asociados a este.
} 
Políticas Sociales (en adelante, GOGEPS) dependiente del Ministerio de Desarrollo Social, el Programa CP cuenta con 49092 hogares beneficiarios integrados por 170824 personas. El $36,7 \%$ de los hogares se encuentra en situación de indigencia y el $63,3 \%$ restante en situación de pobreza.

En relación con la distribución geográfica de los beneficiarios en la Ciudad de Buenos Aires, ${ }^{9}$ un mapeo realizado por la Unidad de Información, Monitoreo y Evaluación (UIMyE, 2011) en base al padrón de beneficiarios del programa muestra la mayor penetración en la zona sur y este de la ciudad, siendo las comunas 7, 8, 4 y 1 las que concentran la mayor parte de la población beneficiaria. ${ }^{10}$ Ello se debe a que dichas comunas están entre las cinco con mayor incidencia de la pobreza en hogares (comuna 7: $8,8 \%$; comuna 8 : $17,1 \%$; comuna 4 : 7,8\%; comuna 1: 9,3\% y comuna 9: $10,8 \%) .{ }^{11}$ Estos valores se ven reforzados por el hecho de que la comuna 8 es la región con mayor cantidad de barrios o asentamientos urbanos denominados como villas, asentamientos y núcleos habitacionales transitorios.

En los hechos, el programa se destina principalmente a las mujeres. Según el Informe de Monitoreo realizado en mayo del corriente año por la GOGEPS, nueve de cada diez transferencias tienen titularidad femenina. En este punto, interesa resaltar que este tipo de políticas sociales establecen a priori la titularidad femenina, situación vinculada al rol otorgado a las mujeres como madres, administradoras del ingreso recibido, organizadoras del cuidado y principales responsables de llevar a cabo las contraprestaciones que exigen los programas. "Esto implica no solo responsabilizarse por el cobro, sino también por la administración burocrática para acceder al beneficio y mantenerlo" (Chahbenderian, 2014: 23).

Las titulares del subsidio conforman una población joven, casi dos de cada tres titulares tiene menos de 45 años. El $70 \%$ de las mujeres titulares cuenta con menos de 12 años de educación formal, y un 30\% ha completado la educación secundaria.

En cuanto a la composición de los hogares, el tamaño promedio de los hogares beneficiarios es de 3,7 miembros, siendo el 38\% del total de beneficiarios menores de 18 años. Casi siete de cada diez hogares cuentan con la presencia de menores de hasta 18 años entre sus miembros. Alrededor del 33\% de las personas se encuentra en edad escolar obligatoria (5 a 18 años).

\footnotetext{
${ }^{9}$ La ciudad se subdivide en 48 barrios, los cuales se agrupan en Unidades de gestión política y administrativa con competencia territorial, llamadas comunas (15).

${ }^{10}$ Haciendo referencia a las comunas señaladas en este artículo, se indican los barrios que las conforman. La comuna 1 está compuesta por Retiro, San Nicolás, Puerto Madero, San Telmo, Monserrat y Constitución; la comuna 4 la conforman los barrios de La Boca, Barracas, Parque Patricios y Nueva Pompeya; la comuna 7 la integran Flores y Parque Chacabuco y por último la comuna 8 abarca los barrios Villa Soldati, Villa Riachuelo y Villa Lugano.

${ }^{11}$ Con respecto a los niveles de pobreza se tuvieron en cuenta las cifras de la EAH 2009, año en que se muestra la última actualización por comunas. Fuente: Incidencia de la pobreza en hogares según INDEC (2009).
} 
Con respecto al lugar de residencia, casi el $60 \%$ de los hogares beneficiarios vive en una villa o asentamiento, en inquilinatos o conventillos, en hoteles o pensiones, en locales no destinados a la vivienda, instituciones colectivas o en situación de calle.

En relación con la inserción laboral, de los hogares pobres o en situación de vulnerabilidad social inscritos al programa, el 34,6 \% está compuesto por al menos un miembro con empleo registrado, jubilado o pensionado -con ingresos que no llegan a cubrir las necesidades básicas el hogar-, mientras que un $65,6 \%$ de los hogares no cuenta con ningún miembro con empleo registrado, jubilado o pensionado (EAHB, Encuesta a Beneficiarios de PCP, 2011). ${ }^{12}$

De acuerdo a los datos arrojados por la Encuesta a Beneficiarios en 2011, el 29\% de las/os titulares de CP son nativos de la ciudad, el $41 \%$ ha llegado desde otros países -el peso de los migrantes extranjeros en el total de la población es considerablemente menor al peso que tienen dentro de los hogares beneficiarios-, y el resto proviene de diferentes provincias argentinas. Cabe mencionar que los titulares nacidos en la provincia de Buenos Aires solo representan el $6 \%$ del total. Entre los extranjeros hay predominio de nativos de países limítrofes y de Perú.

Proceso de inscripción al programa $\mathrm{CP}$, atención y seguimiento

Conforme a la información brindada en la página web C.A.B.A., se realizaron dos operativos de inscripción espontánea: uno entre el 28 de noviembre y 23 de diciembre de 2005, y el otro desde el 20 de marzo al 3 de abril de 2006, difundidos a través de los CGP comunales, los medios de comunicación y otros canales institucionales de información. La inscripción se realizó de forma presencial con la solicitud de la documentación obligatoria anteriormente mencionada para obtener la titularidad del beneficio.

Luego, el programa condujo algunos operativos puntuales y focalizados de inscripción. Para la fecha de realización de la presente investigación, para inscribirse al CP primero debía realizarse la inscripción a Ticket Social (2008) ${ }^{13}$ para luego, evaluación de por medio, ser migrado a CP.

\footnotetext{
${ }^{12}$ La encuesta fue diseñada por la Unidad de Información, Monitoreo y Evaluación y el trabajo de campo fue realizado por la Dirección General de Estadísticas de la Ciudad de Buenos Aires. El universo de análisis fueron los hogares beneficiarios del Programa Ciudadanía Porteña con una muestra de tipo simple al azar a partir de la cual se relevaron 1506 hogares, conformados por un total de 5823 individuos.

13 Ticket Social es un Programa de apoyo alimentario creado en el año 2008 y destinado a fortalecer el consumo de alimentos de las familias que se encuentran en situación de inseguridad alimentaria. Este reemplazó al Programa de Asistencia Alimentaria Directa a Familias, que distribuía módulos alimentarios o cajas de alimentos en forma mensual. Según lo indica el Decreto No 800/008 la asignación se entrega mensualmente y tiene una duración de seis meses que puede ser renovable. El monto mensual es de $\$ 250$ que pueden canjearse por alimentos y elementos de higiene y limpieza en supermercados y comercios adheridos. Para cada integrante de la familia afectado por desnutrición se recibe una chequera de $\$ 130$ y en el caso de celiaquía las madres reciben chequeras por un valor de $\$ 200$ adicionales. La mayor diferencia con respecto a $\mathrm{CP}$ y $\mathrm{AUH}$ es que Ticket Social no impone condicionalidades en materia de salud y
} 


\section{Mecanismos de selección de los beneficiarios}

Al ser el CP un programa asistencial con una población objetivo determinada hogares viviendo en situación de pobreza- requiere de un proceso de identificación y selección.

Según lo indicado en el documento metodológico diseñado por Chitarroni, Novacovsky, y Wermus (2009), el mecanismo de selección de la población beneficiaria del programa consta de tres fases. Una primera etapa de inscripción al Registro Único de Beneficiarios de programas sociales $(R U B)^{14}$ en el que se realiza una ficha de caracterización socioeconómica de los hogares y se entrecruza con otras bases de registro que proveen información sobre inmuebles, automotores, programas sociales, empleo dependiente e independiente, jubilaciones y pensiones, seguro de desempleo, entre otros. Esta primera fase es caracterizada como test de medios directos o test de riqueza.

En una segunda etapa, se realiza un test de medios indirectos que se basa en la estimación de los ingresos potenciales del hogar, calculada a partir de sus características no monetarias, aquellas directamente observables y relativamente más estructurales y estables. Con esta información se constituye un Índice de Vulnerabilidad. Por último, se aplican factores de corrección para evitar errores de inclusión y exclusión.

\section{Modalidad y Monto del beneficio}

El beneficio que otorga el programa consiste en un subsidio mensual que se entrega a través de una tarjeta magnética precargada emitida por el Banco de la Ciudad y Cabal, que debe ser utilizada únicamente para la adquisición de alimentos, productos de limpieza e higiene personal, útiles escolares y combustible para cocinar, que se pueden comprar en los comercios adheridos en la C.A.B.A. como supermercados Día, Coto, carnicerías, verdulerías y almacenes de barrio.

El monto del subsidio ${ }^{15}$ se establece de acuerdo al valor de la canasta básica alimentaria (CBA) tomando como parámetro el adulto equivalente fijado por el Instituto

\footnotetext{
educación a los niños y niñas, y que los y las migrantes también pueden acceder al beneficio presentando su documento extranjero.

${ }^{14}$ La ficha RUB (Registro Único de Beneficiarios) es un instrumento, dependiente de la Gerencia Operativa de Gestión Estratégica de Políticas Sociales (GOGEPS) del Ministerio de Desarrollo Social de la Ciudad Autónoma de Buenos Aires, responsable de censar a los aspirantes de Programas Sociales de la Jurisdicción.

${ }^{15}$ Fecha de relevamiento de la información: marzo de 2015.
} 
Nacional de Estadística y Censos (INDEC), la situación de pobreza del hogar y su composición. Cabe resaltar que el programa utiliza la categoría 'hogar', en lugar de 'familia', y lo circunscribe al grupo de personas, tengan o no relaciones de parentesco entre sí, que viven bajo un mismo techo en forma diaria y permanente, compartiendo sus gastos de alimentación y de sostenimiento.

La actualización de la prestación se realiza semestralmente en función de la variación de la canasta básica alimentaria (corregida en base al índice de inflación calculado por el Congreso Nacional, y otras entidades).

El monto mínimo fijado por hogar es de \$445. Por cada menor de hasta 18 años que integre el hogar se incrementan $\$ 445$, y $\$ 640$ por cada embarazada y niños de cero a dos años. ${ }^{16}$

El monto promedio a cobrar es de $\$ 1000$, de acuerdo al Informe de Monitoreo Ciudadanía Porteña realizado en mayo de 2015 por la GOGEPS, dependiente del Ministerio de Desarrollo Social, siendo el valor mínimo de $\$ 445$ y un máximo de $\$ 2900$. El importe promedio para los hogares en situación de indigencia es de \$1237, mientras que para los que están en situación de pobreza es de $\$ 862$. El monto se calcula de acuerdo a si califican como pobres o indigentes: a los pobres se les paga el $50 \%$ de la canasta alimentaria y a los indigentes el $75 \%$.

La prestación es incompatible con la titularidad de otro programa de transferencias monetarias.

\section{Obligaciones en salud y educación ${ }^{17}$}

Las teorías del capital humano influyeron y conformaron una parte fundamental del diseño de los PTCl, estableciendo condicionalidades de acceso y permanencia. Se puede pensar, siguiendo a Cena (2014) como una responsabilización e individualización del riesgo, al focalizar en el aumento del 'capital humano' la clave para modificar e interrumpir la reproducción intergeneracional de la pobreza.

En sintonía con ello, se observa en los objetivos del programa CP una preocupación explicita por aumentar el 'capital humano', a través de las condicionalidades en salud y educación.

En materia de protección de la salud, CP plantea como uno de sus objetivos principales incrementar la prevención entre los beneficiarios, para lo cual exige la realización de determinados controles de salud.

\footnotetext{
${ }^{16}$ La Ley 2408/2007 aclara que en todos los casos en los que los hogares tengan hijos de hasta dos años, se considerará como adulto equivalente por cada uno de los hijos en esta franja etaria un monto que no podrá ser inferior al establecido en la ley de asignaciones familiares (Ley $N^{\circ} 24714$, Art. 18, Inc. a).

${ }^{17}$ Los hogares sin hijos e hijas a cargo se encuentran exentos del cumplimiento de las condicionalidades, en la medida que estas se restringen a obligaciones relativas al cuidado de los niños, niñas y adolescentes.
} 
De acuerdo al artículo diez de la Ley 1878, los titulares del beneficio asumen las siguientes corresponsabilidades en materia de protección de la salud: controles mensuales durante el embarazo, controles de 'niño sano' y desarrollo nutricional desde el nacimiento hasta los dieciocho años (18) y controles de salud anual para los adultos mayores a sesenta y cinco (65) años de edad y personas con necesidades especiales según periodos estipulados por el Ministerio de Salud de la C.A.B.A. En todos los casos, se debe cumplir con la aplicación de las vacunas obligatorias.

En materia de educación, los principales objetivos planteados por CP son: garantizar la escolarización, reducir la deserción y cooperar en la tarea de erradicar el trabajo infantil. En este sentido, los titulares del beneficio deben procurar que niños/as entre tres y cuatro años asistan al jardín de infantes, presentar certificados cada tres meses de asistencia y permanencia escolar de niños/as de 5 a 18 años (y la promoción al año siguiente de los de 6 a 18 años).

Para comprobar el cumplimiento de los compromisos el Área de Contraprestaciones en Salud y Educación del Programa cruza datos con escuelas e instituciones de salud y se solicita a los hogares beneficiarios la presentación de certificación que avale dicho cumplimiento (Cruces, Epele y Guardia, 2008). Si los hogares no cumplen con los compromisos establecidos, acorde a lo establecido en el Decreto 249/2014-Reglamentación de la ley 1878, se lleva adelante instancias de mediación y seguimiento, suspensión transitoria y, por último, la baja. ${ }^{18}$

En el proceso de información, cumplimiento y control del requisito de salud se cruzan las acciones del Ministerio de Desarrollo Social, del Ministerio de Educación y del Ministerio de Salud de C.A.B.A promoviendo una interrelación entre espacios sociales de acción: el campo económico, el campo de las políticas sociales, el campo del sistema educativo y el de la salud.

\section{Subprogramas complementos del CP}

Desde marzo de 2008 comenzó a implementarse el subprograma Estudiar es Trabajar, un subsidio de $\$ 530$ mensuales para jóvenes estudiantes entre 18 y 40 años en situación de vulnerabilidad social, para que vuelvan a estudiar o continúen sus estudios. El monto es adicional al que reciben sus hogares inscriptos al programa CP. El

\footnotetext{
18 El Decreto 249/2014 establece que en el caso de adeudar algún tipo de documentación, se contempla un plazo improrrogable de seis (6) meses a fin de que el hogar regularice su situación documental. En ese sentido, explicita que previamente y durante dicho proceso se tramitan instancias de mediación y seguimiento a los hogares beneficiarios con el objetivo de identificar y remover los obstáculos que pudieran dificultar o limitar el cumplimiento de los compromisos. Asimismo, se establece que, en el caso de continuar con el incumplimiento, y transcurrido los seis (6) meses mencionados, el programa Ciudadanía Porteña. Con Todo Derecho podrá suspender transitoriamente el beneficio hasta tanto el hogar cumpla con los compromisos correspondientes.
} 
cumplimiento de sus compromisos en educación es condición para su permanencia en el programa (más de la mitad de los titulares del programa asisten al nivel terciario o universitario).

Estudiar es Trabajar se destina a 2536 jóvenes, de acuerdo a la información relevada en mayo de 2015 por la GOGEPS. De acuerdo al informe señalado anteriormente, casi seis de cada diez jóvenes beneficiarios tienen entre 18 y 21 años. Asimismo, seis de cada diez jóvenes beneficiarios son mujeres.

Por su parte, en el año 2013, se dio inicio al subprograma Red Primeros Meses, el cual definió como objetivo acompañar a las madres desde el embarazo y a los chicos recién nacidos hasta el primer año de vida con el fin de detectar y prevenir complicaciones de bajo peso, corta gestación o dificultades respiratorias y así promover el desarrollo de embarazos y bebés saludables.

Se dirige a mujeres de los hogares que se encuentran bajo cobertura de CP. Para adquirir el subsidio las beneficiaras deben completar un cronograma de controles médicos indicados a las mujeres embarazadas y controles en el primer año de vida del bebé.

Durante el embarazo tienen que realizarse el primer chequeo médico dentro de las primeras dieciséis semanas de gestación; luego, dos controles hasta la semana veinticinco y otras dos visitas al médico hasta el final del embarazo. El subsidio es de $\$ 1950$, dividido en tres pagos de $\$ 650$.

Una vez nacido el niño, y para poder obtener el subsidio de $\$ 3000$, el bebé deberá contar con dos controles obligatorios entre las 48 horas y los quince días de vida; realizarse un chequeo mensual hasta los seis meses, y una visita semestral al cumplir el año. Los controles son registrados en una libreta que tendrá cada familia. ${ }^{19}$

La inscripción al programa se realiza a través de la Línea 147, en los Centros Zonales Sociales dependientes del Ministerio de Desarrollo Social y en la sede del programa.

La maternidad adolescente es una compleja problemática en el contexto nacional y de la Ciudad de Buenos Aires. De acuerdo a los resultados relevados en la Encuesta a Beneficiarios, la maternidad a edades tempranas es frecuente entre las titulares de CP: el 34\% de ellas ha tenido su primer hijo antes de cumplir los 20 años. El riesgo de vulnerabilidad social que implica la maternidad muy temprana explica la gran proporción de mujeres que han sido madres adolescentes entre las beneficiarias del programa.

\footnotetext{
${ }^{19}$ Las sumas monetarias señaladas son las informadas en la página web de Ciudad de Buenos Aires en el mes de marzo de 2015: http://www.buenosaires.gob.ar/noticias/un-programa-social-para-madres-derecien-nacidos-y-embarazadas
} 


\section{Impacto sobre indicadores sociales y educación}

El Área de Monitoreo y Evaluación dependiente de la Subsecretaria de Administración del Ministerio de Desarrollo Social ha realizado tres evaluaciones del Programa Ciudadanía Porteña. La última presenta el análisis de los resultados e impactos de CP para el año 2011, a casi seis años de la implementación. ${ }^{20}$ En este apartado se retomarán los aportes de la evaluación realizada y se procederá a su sistematización, considerando que el análisis de los impactos implica leer los datos con cierta cautela, ya que es metodológicamente dificultoso poder aislar el efecto de CP respecto de otras variables de contexto, que pueden estar explicando variaciones en la incidencia de la pobreza o la indigencia, así como en la tasa de asistencia escolar o diversos indicadores en el campo de la salud (Asesoría General Tutelar, 2011).

En relación con las condiciones de vida, de acuerdo a la información oficial en base a estimaciones presentadas, si no existiera el ingreso transferido por el programa, la incidencia de la indigencia en 2011 en niños y adolescentes beneficiarios sería siete veces mayor.

En materia de trabajo infantil y adolescente, la cantidad de niños y adolescentes de 5 a 17 años que trabajan disminuyó un 38\% en el periodo 2006-2011.

En relación con los impactos en educación, la cantidad de niños de tres y cuatro años que asisten a establecimientos de educación formal aumentó un 23,8\% en el periodo 2011 con respecto a 2005. Entre los niños de 6 a 14 años, se mantiene la tendencia a la universalización de la educación.

Las mejoras en los niveles de asistencia resultan considerables entre los adolescentes beneficiarios de 15 a 17 años, donde la deserción disminuyó un $42 \%$ con respecto a 2005. Sin embargo, para el año 2011, persiste un porcentaje alto que no concurre a establecimientos educativos (13,3\%).

En cuanto al mercado laboral entre 2006 y 2011 se observa un leve incremento de cuatro puntos porcentuales en los niveles de actividad de la población mayor de 18 años beneficiaria del programa Ciudadanía Porteña. En 2011, la tasa de actividad se posicionaba en 73,2\%, valor que se ha incrementado desde el año 2008 en 2,3 puntos porcentuales.

Desde una mirada de género, el comportamiento del mercado de trabajo presenta diferencias significativas. Dentro del universo de beneficiarios del $\mathrm{CP}$, los trabajadores masculinos registran una mayor participación en el mercado laboral que sus pares femeninos, la brecha entre ambos es casi de 15,4 puntos porcentuales en el año 2011. Esta tendencia se observa también para el total de la ciudad, donde la brecha entre varones y mujeres es de 19,5 puntos porcentuales. Si se analiza el total de

20 En el año 2011 se diseñó la Tercera Encuesta a Hogares Beneficiarios. La Tercera Evaluación presenta el análisis comparativo entre las tres encuestas realizadas entre 2005 y 2011. 
población en situación de pobreza de C.A.B.A., las diferencias entre las tasas de actividad de varones y mujeres es aún mayor (29,8 puntos porcentuales).

\section{Impacto sobre indicadores de salud ${ }^{21}$}

En primer lugar, el análisis de la población beneficiaria según cobertura de salud muestra que un elevado porcentaje (80\%) no se encuentra afiliada a ningún sistema de salud, dependiendo exclusivamente del sistema público.

En relación con los indicadores de salud, no se advierten mejoras sustantivas en la situación de mortalidad infantil. Según información de la Dirección General de Estadísticas y Censos de la C.A.B.A, la tasa de mortalidad infantil se ha mantenido estable desde la implementación del $\mathrm{CP}$, e incluso ha crecido de 7,8 por mil nacidos vivos en el año 2005 a 8,9 en el año 2013. ${ }^{22}$

En cuanto a los controles durante el embarazo, el $81,6 \%$ de las beneficiarias realiza controles antes del primer trimestre de embarazo. De la misma forma, nueve de cada diez embarazadas realiza controles mensuales. Sin embargo, el porcentaje de embarazadas que realiza controles tempranos descendió en tres puntos porcentuales entre 2008 y 2011, mientras que el porcentaje de embarazadas que realiza controles mensuales de embarazo se mantuvo constante en los dos periodos.

En el caso de los menores de dos años, un alto porcentaje de las madres ha realizado el control de niño sano antes del primer mes $(87,7 \%)$ y la mayor parte de las madres ha realizado controles a su hijo antes del tercer mes. En los tres momentos analizados, se observa que más del $97 \%$ de las madres realiza controles de salud antes de que sus hijos cumplan los tres meses de vida. Cabe señalar que el porcentaje de mujeres que realiza controles periódicos de salud a su hijo menor de dos años disminuye un $7 \%$ en el período analizado (2011). Asimismo, no se observa una mejora en el porcentaje de mujeres que realizan controles de salud antes del primer mes de vida. A raíz de los valores registrados para estos indicadores el informe resultante de la Tercera Evaluación indica que estas situaciones señalan un déficit en la exigencia de certificados de salud, volviendo a poner en escena la idea de una autorresponsabilización del individuo. En este sentido, y siguiendo la línea planteada por Cena (2014) este tipo de programas no hacen referencia a las condiciones estructurales que, en tanto complejos entramados causales, desembocaron en esas situaciones de carencias.

\footnotetext{
${ }^{21}$ Fuente: Encuestas a Hogares Beneficiarios 2006; 2009 y 2011-DGEyC.

22 La Tasas de mortalidad infantil total, neonatal, postneonatal y fetal (por mil nacidos vivos). Ciudad de Buenos Aires. Años 1990/2013. Dirección General de Estadísticas y Censos, Ministerio de Hacienda. Estadísticas vitales.
} 


\section{Reflexiones finales}

Realizar este breve recorrido por el programa CP se planteó como un intento de aportar a su problematización, dando cuenta de sus características, alcances, coberturas, las condiciones de vida de la población destinataria y también los impactos de la política pública, tras una década de implementación.

La presente caracterización realizada a partir de la recopilación de información oficial que brinda el gobierno de C.A.B.A sobre el programa, permite echar luz sobre las formas y modalidades que adquieren a partir de la década de 90 las políticas sociales, periodo en el cual la protección social deja de estar estructurada en función del empleo formal. Asimismo, también posibilita explorar la identidad específica de la política social, es decir, las particularidades que se configuran alrededor del diseño e implementación del Programa de Transferencias Condicionadas de Ingresos Ciudadanía Porteña-Con todo derecho.

Luego de la descripción realizada se puede identificar a CP como un PTCl, dado que contiene las características fundamentales de este tipo de programas. En primer lugar, transfiere ingresos a una población específica -exclusivamente para la adquisición de productos alimentarios y demás elementos indispensables para el hogar-; en segundo lugar, establece condicionalidades para poder percibir el ingreso, en forma de asistencia escolar y atención de la salud, con el objetivo de contribuir al fortalecimiento del 'capital humano'.

$\mathrm{CP}$ se define como una política social que tiene como objetivo principal cortar la reproducción intergeneracional de la pobreza. Para ello, establece situaciones prioritarias de pobreza, al indicar una población objetivo, criterios de selectividad y construcción de un índice de vulnerabilidad.

Es un programa de carácter focalizado que se ha configurado como masivo, dado su alcance en la C.A.B.A. Como señala De Sena "Estos programas están dirigidos a un tipo de población particular, con alguna determinada característica, dejando en evidencia su focalización y la atención a un efecto de la problemática y no a su causa" (De Sena, 2011: 56). La autora nos propone pensar en este tipo de intervenciones vinculadas al concepto de masividad. Es así como se las denomina políticas de inclusión masiva, pero no universales, un tipo de políticas focalizadas que presentan particularidad en el número de personas que gozan del beneficio (De Sena, 2014).

Por último, luego de diez años de implementación del programa, cobra relevancia preguntarnos si este tipo de intervenciones públicas sirven para cortar la reproducción intergeneracional de la pobreza. Como así también, reflexionar acerca del cumplimiento de los objetivos que define $\mathrm{CP}$ relacionados con promover el acceso a la educación y la salud, especialmente en los niños, niñas y adolescentes. Ello lleva a pensar el lugar de las contraprestaciones en este tipo de políticas sociales, y permite, 
por último, abrir algunos interrogantes al respecto: ¿qué relaciones se construyen entre los beneficiarios con los campos de la educación y la salud en este tipo de programas que exigen la presentación de contraprestaciones? ¿qué prácticas sociales se configuran? ¿es posible pensar en un fortalecimiento del 'capital humano' mediante transferencias monetarias? Quedan planteados estos interrogantes para futuras investigaciones.

\section{Bibliografía}

Área Monitoreo y Evaluación, Subsecretaría de Administración. Ministerio de Desarrollo Social GCBA (2011). Resultados de la tercera evaluación del programa Ciudadanía Porteña y del componente Estudiar es trabajar. [fecha de consulta: 8 Julio 2015]. Recuperado

http://www.buenosaires.gob.ar/sites/gcaba/files/tercera evaluacion de ciudadania port ena.pdf

Asesoría General Tutelar (2011). Programa Ciudadanía Porteña ¿con todo derecho?: Fortalezas y debilidades de las transferencias monetarias condicionadas focalizadas en niños, niñas y adolescentes. -1ra ed.- Buenos Aires: Eudeba. Recuperado de: www.asesoria.jusbaires.gov.ar

AZPIAZU, D. y BASUALDO, E. (2004). Las privatizaciones en la Argentina. Génesis, desarrollo y principales impactos estructurales. En publicación: Las privatizaciones en la Argentina. Génesis, desarrollo y principales impactos estructurales. FLACSO, Facultad Latinoamericana de Ciencias Sociales, Sede Argentina. Disponible en: http://bibliotecavirtual.clacso.org.ar/ar/libros/argentina/flacso/azpiazu.pdf

BARZANI, C., DOMINGUEZ, I., GIANNI, C., KUPERMAN, V. y LAGO, N. (2001): Proyecto: prevención primaria y secundaria en $\mathrm{VIH}$-sida en Salud y Población, Cuadernos de capacitación de la Residencia Interdisciplinaria de Educación para la Salud, N², Buenos Aires: Dirección de Capacitación GCBA.

BOURDIEU, P. (1988). Espacio social y poder simbólico. En Cosas dichas. Buenos Aires: Gedisa.

CASTILLA, M. (2014). Maternidad y política social: experiencias y sentidos atribuidos a los ingresos monetarios percibidos por el programa Ciudadanía Porteña, Buenos Aires. Población \& Sociedad [en línea], ISSN-L 0328 3445, Vol. 21 (1), 2014, pp. 41. Disponible en: http://www.poblacionysociedad.org.ar/archivos/21/P\&S-V21-N1-Castilla.pdf

CECCHINI, S. (2011). Protección Social y programas no contributivos en América Latina. Seminario Internacional "Sistema de Protección Integral", Quito: División de Desarrollo Social Comisión Económica para América Latina y el Caribe (CEPAL).

Disponible en: http://www.cepal.org/dds/noticias/paginas/6/28106/protsocnocontr.pdf CECCHINI, S y MADARIAGA, A. (2011) Programas de Transferencias Condicionadas: balance de la experiencia reciente en América Latina y el Caribe. Cuadernos de la 
CEPAL.

Disponible

en:

http://www.cepal.org/dds/noticias/paginas/6/28106/protsocnocontr.pdf

CENA, R. (2014). Programas de transferencias condicionadas de ingresos y programas de empleo en Argentina: entre la responsabilización de los destinatarios y la individualización de la cuestión social. Boletín Científico Sapiens Research, 4(1), 3-8.

CEPAL (2014). Base de datos de programas de protección social no contributiva. División de Desarrollo Social. Recuperado de: http://dds.cepal.org/bdptc

CHAHBENDERIAN, F. (2015). ¿Cómo se construye la población destinataria desde los Programas de Transferencias Monetarias Condicionadas? Nuevas formas de abordaje de la "cuestión social" basadas en la autorresponsabilización. Boletín Científico Sapiens Research Vol. 5, 19-24. Disponible en:

http://www.sapiensresearch.org/images/pdf/v5n1/V5N1 Sociologando 1.pdf

CHITARRONI H., NOVACOVSKY I., y WERMUS N., (2009) Los métodos de identificación y selección de los beneficiarios del Programa Ciudadanía Porteña, GCBA. Disponible en: http://estatico.buenosaires.gov.ar/areas/des social/ciudadania portenia/informes condiciones vida/Metodos de Identificacion y Seleccion Beneficiarios Ciudadania Por tena.pdf

CRUCES, G., EPELE, N., GUARDIA, L. (2008) Los programas sociales y los objetivos de desarrollo del Milenio en Argentina. Chile: División de Desarrollo Social (CEPAL).

Decreto No: 249 (2014) Reglamentación de la Ley No 1878 - Ciudadanía Porteña con Todo Derecho. Ciudad Autónoma de Buenos Aires. Disponible en: http://www.ciudadyderechos.org.ar/reg decretos home.php

Gerencia Operativa de Gestión Estratégica de Políticas Sociales (GOGEPS). Ministerio de Desarrollo Social (2015). Informe de Monitoreo Ciudadanía Porteña mayo 2015. Recuperado

de:

http://www.buenosaires.gob.ar/sites/gcaba/files/informe monitoreo cp -

mayo 2015 0.pdf

DE SENA, A. (2011) "Promoción de Microemprendimientos y políticas sociales: ¿Universalidad, Focalización o Masividad?, una discusión no acabada". En Revista Pensamiento Plural. Año 4 No 8, pp 36-66.

DE SENA, A. (2014) (coord.). Las políticas hecha cuerpo y lo social devenido emoción: lecturas sociológicas de las políticas sociales. Ciudad Autónoma Buenos Aires, Argentina: Estudios Sociológicos Editora/Universitas. Editorial Científica Universitaria. Recuperado de http://estudiosociologicos.org/portal/lecturas-sociologicas-de-las-politicas/

DE SENA, A. Y MONA, A. (2014) A modo de introducción: la cuestión social, las políticas sociales y las emociones. En De Sena, A. (ed.) Las políticas hechas cuerpo y lo social devenido emoción (pp.9-18). Ciudad Autónoma de Buenos Aires. Estudios sociológicos editora. 
Dirección General de Estadística y Censos GCBA, Ministerio de Hacienda. La mortalidad infantil en la Ciudad de Buenos Aires: 2000 - 2011. Informe de resultados. Recuperado de http://www.buenosaires.gob.ar/areas/hacienda/sis estadistico/ir 2012 499.pdf Dirección General de Estadísticas y Censos, Ministerio de Hacienda GCBA. Encuesta Anual de Hogares (EAH) 2011. Recuperado de:

http://www.buenosaires.gob.ar/areas/hacienda/sis estadistico/EAH/encuesta anual ho gares index.php

HERRERA, M. Y CASTÓN, P. (2003). Las políticas sociales en sociedades complejas. Barcelona: Ariel Sociología.

Ley No 1878. Crea el Programa "Ciudadanía Porteña. Con todo derecho". Legislatura de la Ciudad de Buenos Aires, 01 de diciembre de 2005 - Artículo 2 y 4 . Recuperado de http://www.cedom.gov.ar/es/legislacion/normas/leyes/anexos/drl1878.html

PUELLO SOCORRÁS, J. Y GUNTURIZ, M. (2013). ¿Social-neoliberalismo? Organismos multilaterales, crisis global y programas de transferencia monetaria condicionada. México: Universidad Autónoma Metropolitana. N40 versión impresa ISSN 0188-7742 Unidad de Información, Monitoreo y Evaluación (UIMyE) Ministerio de Desarrollo Social, Gobierno de la Ciudad de Buenos Aires (2011). Mapas de la pobreza y los programas en el terriotorio. Recuperado de: http://estatico.buenosaires.gov.ar/areas/des social/evaluacion programas/informes condiciones vida/mapasall 2011.pdf VILAS, C. (1995) (coord.) Estado y políticas sociales después del ajuste. Caracas: Nueva Sociedad/Universidad Nacional Autónoma de México.

WEB del Gobierno de la Ciudad de Buenos Aires:

http://www.buenosaires.gob.ar/areas/des social/ciudadania portenia 